\title{
On research of optimal programming solutions for road construction in modern conditions
}

\author{
Tatiana Dormidontova ${ }^{1 *}$, Sergey Evdokimov ${ }^{1}$, and Natalia Shekhova ${ }^{1}$ \\ ${ }^{1}$ Samara State Technical University, Institute of Architecture and Civil Engineering, \\ Molodogvardeyskaya str. 194, 443001 Samara, Russia
}

\begin{abstract}
The poor condition of roads in most regions of Russia predetermines the need to establish and implement target regional programs of road network development in the country. Depending on the time perspective of the mentioned programs (short, medium or long term), the defining criteria for including roadways in these programs are set. To develop the theory and practice of justification of optimal programming solutions in road construction in the context of multi-criteria assessment, the authors have proposed the method to optimize the programming solutions in road construction and the algorithm of its implementation. The basic principles of comparing alternative variants for the construction of roadways with comparable pavement design as well as recommendations on the selection of the optimal variant have been developed using the technical and economic indexes.
\end{abstract}

\section{Introduction}

In many regions of the country the state of roadways could be described as unsatisfactory. In this regard, the implementation of regional target programs of the road network development is currently gathering pace [1-3].

The purpose of the work is to provide the optimal programming solutions for road construction in the context of multi-criteria assessment.

The objectives of the research are:

- to identify the criteria for including roads in the short-, medium- and long-term regional target programs;

- to develop the method for optimizing programming solutions in road construction and the algorithm of its implementation;

- to select the optimal variant of the construction of roadways with comparable pavement designs.

\section{Main Part}

The in-depth analysis of the roadways condition allowed identifying characteristic criteria for including them in relevant programs of roads development. When creating these

* Corresponding author: adisk63@yandex.ru 
programs it is necessary to take into account such factors as completion schedules and the importance of work for road development. Depending on what time perspective these programs are oriented on, they are united into three groups: short-, medium- and long-term ones [4-7].

The main criteria for including roads in the operational program of development are the following:

- the number of road sections with high concentration of accidents that have occurred due to adverse road conditions;

- the number of road sections passing through populated areas with high intensity of transit traffic;

- road sections which are school bus routes that do not meet regulatory requirements.

The fundamental criteria for including roads in the medium-term development program are:

- non-compliance of the actual bridge clearance to regulatory requirements;

- road sections with expired intermaintenance period;

- unfinished objects;

- road sections, which are school bus routes.

The defining criteria for including roads in the long-term development program are the following indexes:

- crossing of a road and a railway at the same level;

- existing transport corridors and prospective strategy of their development;

- municipal streets (in urban areas), which are a continuation of public roads;

- rural settlements that do not have hard surface driveways;

- rural settlements with a high density of transit traffic;

- road sections, where traffic density exceeds the norm for this category.

For the development of the theory and practice of providing optimal programming solutions in road construction in the context of multi-criteria assessment, the following method has been proposed by the authors. The algorithm of its implementation is described below [8-10].

The variety of factors raises difficulties when selecting specific areas of work and prioritizing their implementation. That is why, it is proposed to introduce the point assessment of each factor influence. Based on this assessment system, one can determine the significance of an object in points, which consequently makes it possible to form a ranged list of those objects. This list shows the order of work performance to achieve a multiplier effect.

It is necessary to take into account the factors that require consideration in order to achieve socio-economic effects, bearing in mind the immensity of the tasks.

For example, such criterion as "Connection of a rural settlement with population of 100 people with the existing network of public roads by means of a road" gets one point, similar criterion "a settlement with population of 500 people" - three points. The criterion: "Construction of a roadway up to $1 \mathrm{~km}$ " - three points, the criterion for "Construction of a roadway up to $5 \mathrm{~km}$ " - one point. The ranged list of roads is formed according to the total of points in order of descending [11].

The proposed principles give grounds for comparison of alternatives for laying roadways with comparable pavement designs, in order to select the best one. The technical and economic indexes are compared.

The main criteria for comparing the roadways are as follows:

- the minimum radius of the curve in the plan;

- the minimum radius of vertical curves;

- the availability and number of artificial structures;

- the specific index of the earth quantities per $1 \mathrm{~m}^{3}$; 
- the length of a road section with the possible longitudinal slope;

- the payback period;

- the construction cost per $1 \mathrm{~km}$ of the road.

When introducing the points system for each of these parameters, one can perform the comparison which results in the optimal plan for roadway construction.

One of the urgent problems of the modern road construction is to compare alternative pavement designs. The most important problem that should be solved at the final stages of road construction is the selection of variants of the pavement design from the variety of layers which are optimal for a particular area. When selecting the best option, three possible designs of the pavement are considered: asphalt concrete covering; cement concrete covering; cement concrete base with asphalt covering [12-16].

The efficiency indexes serve as the selection criteria. They are presented in Table 1.

Table 1. Indexes of the selection criteria of the road effectiveness.

\begin{tabular}{|l|c|c|c|}
\hline Criteria Indexes & Variant 1 & Variant 2 & Variant 3 \\
\hline Service Life (A) & 15 years & 25 years & 20 years \\
\hline Estimated cost (B) & $\begin{array}{c}10634.6 \text { thousand } \\
\text { rubles. }\end{array}$ & $\begin{array}{c}108468.1 \\
\text { thousand rubles. }\end{array}$ & $\begin{array}{c}27983.0 \\
\text { thousand rubles. }\end{array}$ \\
\hline $\begin{array}{l}\text { The need for machinery } \\
\text { and labor force (C) }\end{array}$ & $103 / 20$ & $133 / 140$ & $127 / 135$ \\
\hline $\begin{array}{l}\text { Utilization level of local road- } \\
\text { building machinery (D) }\end{array}$ & $16 \mathrm{~km}$ & $91 \mathrm{~km}$ & $91 \mathrm{~km}$ \\
\hline Intermaintenance period (E) & 5 years & 12 years & 5 years \\
\hline Maintainability (F) & 0.4 & 0.2 & 0.4 \\
\hline Operational costs (G) & 113.78 & 74.3 & 113.78 \\
\hline Pavement wearing capacity (H) & $1 \div 1.5 \mathrm{~mm} /$ year & $\begin{array}{c}0.1 \div 0.2 \mathrm{~mm} / \\
\text { year }\end{array}$ & $1 \div 1.5 \mathrm{~mm} /$ year \\
\hline
\end{tabular}

In order to define the significance of the particular criteria the relative preference method is used, which is a special case of the expert method. This method is for one expert who is the decision-maker. It includes the following steps.

First. Prioritization of each criterion in the following order:

$$
A(8) \geq B(7) \geq C(6) \geq D(5) \geq E(4) \geq F(3)=G(3) \geq 3
$$

Second. Determination of the weight coefficient for each criterion. Each line at its intersection with the column contains the value, corresponding to the order of preference of the line criterion to the column criterion. As a result, each line gives the sum of the importance coefficient, Table 2.

Table 2. Weight coefficients.

\begin{tabular}{|c|c|c|c|c|c|c|c|c|c|c|}
\hline & $\boldsymbol{A}$ & $\boldsymbol{B}$ & $\boldsymbol{C}$ & $\boldsymbol{D}$ & $\boldsymbol{E}$ & $\boldsymbol{F}$ & $\boldsymbol{G}$ & $\boldsymbol{H}$ & $\sum \beta_{i j}$ & $A_{i}$ \\
\hline$A$ & - & 1 & 2 & 3 & 4 & 5 & 5 & 7 & 27 & 0.34 \\
\hline$B$ & 0 & - & 1 & 2 & 3 & 4 & 4 & 6 & 20 & 0.25 \\
\hline$C$ & 0 & 0 & - & 1 & 2 & 3 & 3 & 5 & 14 & 0.18 \\
\hline$D$ & 0 & 0 & 0 & - & 1 & 2 & 2 & 4 & 9 & 0.11 \\
\hline$E$ & 0 & 0 & 0 & 0 & - & 1 & 1 & 3 & 5 & 0.06 \\
\hline$F$ & 0 & 0 & 0 & 0 & 0 & - & - & 2 & 2 & 0.03 \\
\hline
\end{tabular}




\begin{tabular}{|c|c|c|c|c|c|c|c|c|c|c|}
\hline$G$ & 0 & 0 & 0 & 0 & 0 & - & - & 2 & 2 & 0.03 \\
\hline$H$ & 0 & 0 & 0 & 0 & 0 & 0 & 0 & - & 0 & 0 \\
\hline & & & & & & & & & $\sum 79$ & $\sum 1$ \\
\hline
\end{tabular}

Third. Determination of the standardized values of the partial criteria, Table 3.

Table 3. Standardized values of the partial criteria.

\begin{tabular}{|l|l|l|l|l|l|l|l|c|}
\hline Variants & $A_{H}$ & $B_{H}$ & $C_{H}$ & $D_{H}$ & $E_{H}$ & $F_{H}$ & $G_{H}$ & $H_{H}$ \\
\hline 1 & 1.0 & 0.83 & 0.6 & 0.77 & 0.18 & 0.42 & 1.0 & 1.0 \\
\hline 2 & 0.15 & 0.84 & 1.0 & 1.0 & 1.0 & 1.0 & 0.5 & 0.65 \\
\hline 3 & 1.0 & 1.0 & 0.75 & 0.75 & 1.0 & 0.42 & 1.0 & 1.0 \\
\hline
\end{tabular}

Fourth. The generalized criterion of efficiency is calculated taking into consideration the values of the index and the standardized values of the criteria for each variant. Since all criteria are minimized, the formula (2) is used:

$$
Y_{1}=\sum_{i=1}^{n} \alpha_{i} K_{i}^{H} \rightarrow \min (\max )
$$

Fifth. Determining the best variant, which is the one with the minimum generalized criterion from three given. In the analyzed example, it is the pavement design with asphalt concrete covering. variant.

If you change the importance priority of particular criteria, the best one may be another

\section{Conclusion}

After considering the results of the research the following conclusions have been made:

1. The criteria for including the roads in the short-, medium- and long-term regional target programs have been identified.

2. The method of optimization of programming solutions in road construction and the algorithm of its implementation have been developed. Studies have shown that the quality of the developed method is determined by the algorithm and depends on a set of the analyzed criteria. Despite all the advantages of this method, it should be remembered that in order to build a high-quality model, it is necessary to understand the nature of the relationship between the dependent and independent variables and prepare a sufficient data set to determine the basic criteria for comparison.

3. Because of the unstable economic situation in Russia companies have to make more thorough and balanced decisions, focusing either on the quality of work performed or on the amount of funds allocated for the construction, prepare development plans, based on pilot tests and practical knowledge, evaluating the reality.

\section{References}

1. M.I. Balzannikov, N.V. Ahrameeva, Environmental and hydraulic engineering structures: problems of construction, operation, environment and specialists training: Proceedings of International Scientific and Technical Conference (2014) 
2. M.I. Balzannikov, Yu.M. Galitskova, A. A. Bolotova, Bulletin of the Volga regional branch of the Russian Academy of architecture and construction sciences: Proceedings, 16, 132-135 (2013)

3. V.I. Gvozdovsky, S.V. Evdokimov, Environmental and hydraulic engineering structures: problems of construction, operation, environment and specialists training. Proceedings of International Scientific and Technical Conference, 219-220 (2014)

4. L.G. Goverdovskaya, Environmental and hydraulic engineering structures: problems of construction, operation, environment and specialists training. Proceedings of International Scientific and Technical Conference, (2014)

5. T.V. Dormidontova, N.G. Solkaran, Environmental and hydraulic engineering structures: problems of construction, operation, environment and specialists training. Proceedings of International Scientific and Technical Conference, (2014)

6. T.V. Dormidontova, S.V. Evdokimov, Complex application of methods of reliability assessment and monitoring of structures and buildings, (SGASU, Samara, 2012)

7. T.V. Dormidontova, V.V. Kiryakov, Internet-journal "Science of Science" 7(1), (2015) information on http://www.naukovedenie.ru/ PDF/ 42TVN115.pdf/

8. T.V. Dormidontova, Tradition and Innovation in Civil Engineering and Architecture: Proceedings of the 70th Scientific and Technical Conference on R \& D Results in 2012 (Samara, 2013)

9. T.V. Dormidontova, Procedia Engineering XXIV R-S-P seminar, Theoretical Foundation of Civil Engineering, (2015)

10. E.I. Marinin, T.V. Dormidontova, Environmental safety in construction 4(17), 80-84 (2014)

11. S.V. Evdokimov, B.G. Ivanov, V.A. Shabanov, Scientific review 22, 102-107 (2015)

12. Utility Patent 160415. Protective device for roads, Published 20.03.2016. Bull. № 8

13. N.V. Ovchinnikova, Institutional support of economic mechanism of externalities regulation (Samara, 2010)

14. N.V. Shekhova, I International Research and Practice Conference "Innovative development strategies of economy and management", Proceedings, (2014)

15. N.V. Shekhova, Modern economy: problems, solutions, prospects: Proceedings of International Research and Practice Conference dedicated to the 20th anniversary of Economic Department, 156-160 (2014)

16. N.V.Shekhova, P. Surmakh, II International Research and Practice Conference "Economic aspects of management of a building complex in modern conditions": Proceedings, 68-72 (2014) 